\title{
Power-like corrections to the conductivity of Mo-C nanocomposites
}

\author{
A. D. Bozhko \\ A. M. Prokhorov General Physics Institute, Russian Academy of Sciences, Moscow, Russia \\ bozhko@lt.gpi.ru
}

PACS 72.80.Tm, 72.80.Ng

DOI 10.17586/2220-8054-2016-7-1-169-174

\begin{abstract}
The evolution of the electron transport character in thin films of Mo-C nanocomposites is studied over the metal concentration range $0.14-0.3$ at temperatures from 4.2 to $400 \mathrm{~K}$. It is shown that conductivity of the Mo-C nanocomposites demonstrates the distinct power-like behavior in the two temperature intervals separated at $20-$ $25 \mathrm{~K}$. The concentration dependences of the power exponent in both temperature intervals are characterized by the expressed minimum at Mo content close to 0.2 varying over the range $0.25-1.0$. The experimental data are discussed and simulated in the model of inelastic tunneling of the electrons in amorphous dielectrics in the framework of the effective medium approximation.
\end{abstract}

Keywords: metal-carbon nanocomposite, granular materials, amorphous carbon, electron transport, inelastic tunneling.

Received: 20 November 2015

\section{Introduction}

The interest in nanocomposites is motivated by their potential applications as sensors, optoelectronic devices, etc. [1]. The technological flexibility of the synthesis gives an easy way to generate the desired electrical response of the sensors under external excitation. This greatly broadens the field of application for nanocomposites. On the other hand, the competition among the number of physical phenomena such as grain-to-grain tunneling of the electrons, their quantum confinement on a single grain, Coulomb blockade, discrete energy spectrum of the grains, electron-electron interaction, etc. gives an opportunity to use the nanocomposites as model objects to study the peculiarities of electron transport in granular materials [1]. The metalcarbon nanocomposites are materials of particular importance. They combine the extraordinary mechanical properties typical for the hard forms of amorphous carbons [2] and the functionality of the nanogranular conducting materials [1]. The complex structure of amorphous carbon allows one to study the influence of the enhanced hierarchically-scaled disorder on the electron transport in the nanogranular matter.

\section{Samples preparation and methods used}

The films of molybdenum-carbon nanocomposites with a thickness of $1 \mu \mathrm{m}$ were grown on the polished dielectric substrates in the hybrid deposition setup. The carbon-containing dielectric matrix was deposited by PCVD of siloxane $\left(\left(\mathrm{CH}_{3}\right)_{3} \mathrm{SiO}\left(\mathrm{CH}_{3} \mathrm{C}_{6} \mathrm{H}_{5} \mathrm{SiO}\right)_{3} \mathrm{Si}\left(\mathrm{CH}_{3}\right)_{3}\right)$ vapors in DC plasma discharge. The DC magnetron co-sputtering of Mo target was used for the metal phase generation. The substrates were positioned on the RF-biased (1.84 MHz) substrate holder. The detailed description of the deposition process can be found elsewhere [2]. The carbon phase of the deposited films is amorphous [4] and highly insulating $\left(10^{12}-10^{14} \mathrm{Ohm} \cdot \mathrm{cm}\right.$ [3]). In accordance with TEM data, the molybdenum phase has a granular structure with the 
typical size of amorphous Mo-rich domains about $1.1 \pm 0.3 \mathrm{~nm}$. The peculiarities of the structure allow one to relate the Mo-C nanocomposites to the disordered nanogranular materials.

The DC conductivity of the nanocomposites was measured over $4.2-400 \mathrm{~K}$ by the routine 4-terminal technique in a gas-flow helium cryostat. The samples were patterned in RF (13.56 MHz) Ar discharge by the use of mechanical mask in a double Hall cross configuration. The elemental composition of the molybdenum-carbon nanocomposites was studied by EPMA JEOL JXA-8200.

\section{Results and discussions}

The experimental dependences of the scaled conductivity on temperature $\sigma(T) / \sigma(400)$ are shown in Fig. 1 (a) over the Mo concentration range $0.14-0.3$. The Mo concentration is shown near each curve. It can be seen that conductivity decreases with temperature over the entire range of Mo concentration. In the nanocomposites studied, the transition to the superconducting state is observed. The transition temperature is dependent on the Mo concentration. However, this phenomenon is beyond the scope of this paper.

The $\sigma(T)$ dependences possess the fine structure clearly observed in the temperature dependences of the derivative of the conductivity on temperature. The $\sigma^{\prime}(T) / \sigma^{\prime}(400)$ dependences are shown on a double logarithmic scale in the insert to Fig. 1(a). As it can be seen, the temperature dependences of the conductivity derivative are characterized by the existence of two distinct temperature intervals (marked in the insert to Fig. 1(a) as I and II) separated at temperature $20-25 \mathrm{~K}$. The extended linear parts of $\sigma^{\prime}(T)$ dependences within each temperature interval correspond to the power dependences of the conductivity on temperature:

$$
\sigma(T)=\sigma_{0}+\sigma_{1}\left(\frac{T}{T_{0}}\right)^{p}
$$

where $T_{0}$ is a characteristic temperature.

The principal parameter in the modern models of the electron transport in granular media, which allows one to discriminate the mechanisms of the electron transport, is the value of the power exponent $p$. In the temperature range $g_{T} \delta<k_{B} T<E_{C}$ the corrections to the conductivity of an ordered granular array assume the logarithmic form ( $p$ close to 0$)$ [5]:

$$
\Delta \sigma(T) \sim-\frac{1}{g_{T} d} \ln \left(\frac{g_{T} E_{C}}{k_{B} T}\right),
$$

where $d$ is the dimensionality of the sample, the dimensionless intergranular conductance $g_{T}=$ $G_{T} /\left(2 e^{2} / h\right) \gg 1, \delta=\left(g_{F} b^{3}\right)^{-1}$ - the mean distance between energy levels in a single grain with size $b, k_{B}$ is Boltzmann's constant, and $E_{C} \approx e^{2} / 2 \varepsilon b$ - Coulomb charging energy of a single grain ( $\varepsilon$ is the effective dielectric constant of the media).

At lower temperatures $k_{B} T<g_{T} \delta$, the corrections to the conductivity in a 3-dimensional case accept the power form [6]:

$$
\Delta \sigma(T) \sim-\frac{1}{g_{T}} \sqrt{\frac{k_{B} T}{g_{T} \delta}} .
$$

Figure 1(b) illustrates the concentration dependences of the power exponent $p$ in $\mathrm{Mo}-\mathrm{C}$ nanocomposites obtained by fitting of the conductivity curves (Fig. 1(a)) in temperature intervals I and II using Eq. (1). The power exponent $p$ varies over the range $0.25-1.0$ being systematically higher in temperature interval I. Both dependences are characterized by the minimum at Mo concentration around 0.2. It follows from this figure that: a) the logarithmic corrections to the 
Power-like corrections to the conductivity...

conductivity (2) are not observed in Mo-C films, b) the square root corrections (3) exist only at certain Mo concentration values.

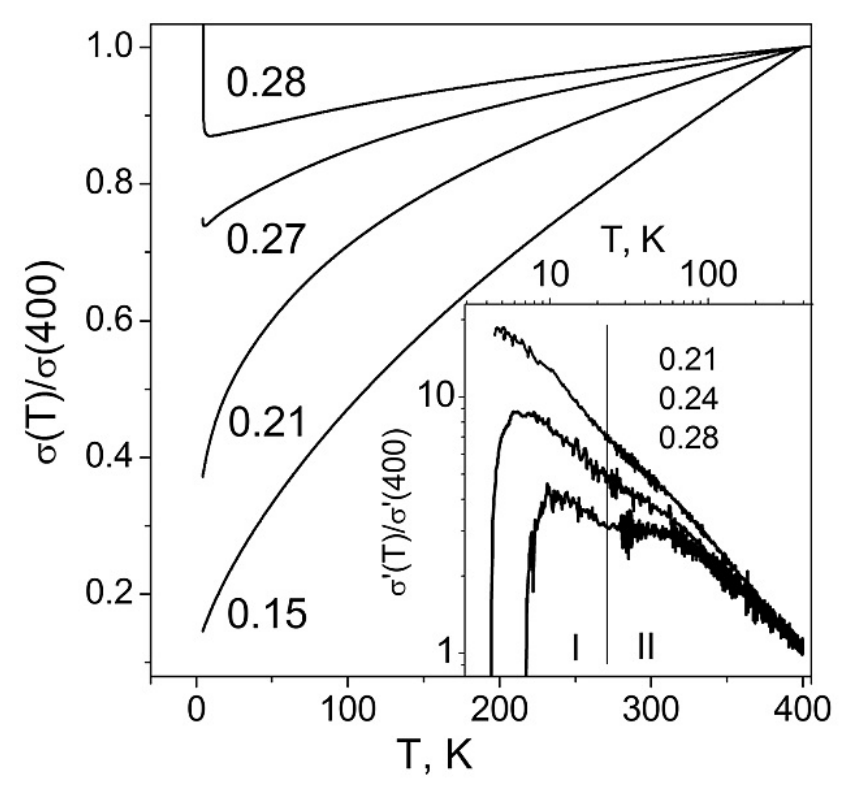

(a)

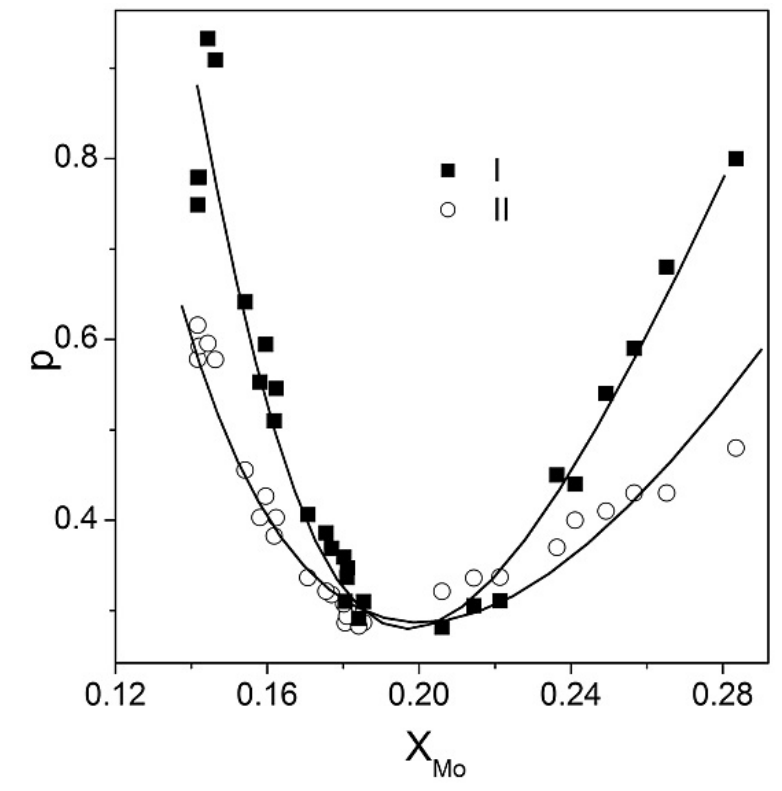

(b)

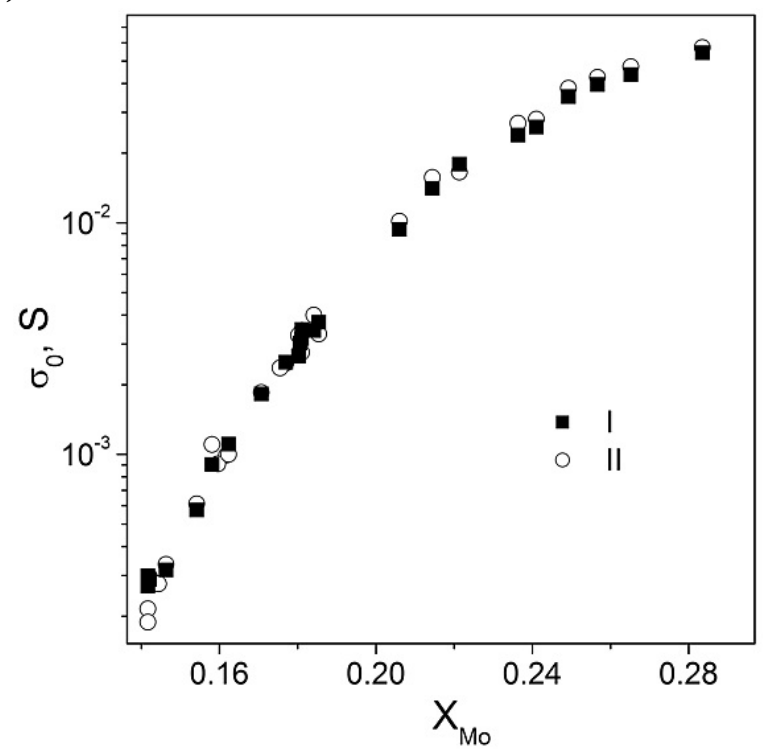

(c)

FIG. 1. (a) Normalized conductivity $\sigma(T) / \sigma(400)$ of Mo-C nanocomposite films $v s$. temperature, insert - normalized derivative $\sigma^{\prime}(T) / \sigma^{\prime}(400) v s$. temperature; (b), (c) Mo concentration dependence of power exponent $p$ (Eq. (1)) (b) and $\sigma_{0}$ (c) of $\sigma(T)$ dependences in temperature intervals I and II; Lines are guide to the eyes only (b). Explanations are given in text

It is generally accepted that the tunneling-percolation model is a good approximation for disordered granular materials [7]. Within this model the charge transfer occurs via tunneling of the electrons between the conducting grains. The temperature dependence of the conductivity of such granular media exists if between at least some conducting grains the temperature-dependent intergrain tunneling takes place. 
The candidate for the role of the temperature-dependent tunneling may be the inelastic intergranular tunneling of the electrons proposed as a main charge transfer mechanism in thin films of amorphous dielectrics [8]. Following this model, the conductivity across the thin amorphous dielectric is formed in the tunnel channels via the chains of localized states. The conductivity of the single chain containing more than two localized states $n$ depends on the temperature by the power way:

$$
\langle\sigma\rangle_{n} \sim S e^{2}\left(\frac{\Lambda^{2}}{\rho c^{5}}\right)^{\frac{n-1}{n+1}} g^{n} n^{2 n} a^{2 n-1} l^{n-1} E_{0}^{\frac{2 n}{n+1}} T^{n-\frac{2}{n+1}} \exp \left(-\frac{2 l}{a(n+1)}\right),
$$

where $l$ is a distance between electrodes, $S$ - cross-section area of the sample, $\Lambda$ - deformation potential constant, $\rho$ - density of matrix material, $c$ - velocity of the sound in the matrix, $g-$ density of localized states at the Fermi level, $a$ - radius of the localized state, and $E_{0}$ is the depth of localized states inside the barrier.

The total conductance of a sample is the sum of the conductance of all the channels. The model of the inelastic tunneling of the electrons gives a good basis for understanding the peculiarities of electron transport in disordered granular materials. In accordance with it, above the metal insulator transition point, the infinite conducting cluster, which consists of tunnel junctions with not more than one localized state in the intergrain potential barriers, exists. The temperature dependence of the conductivity of such a cluster is weak [8]. The parts of the infinite conducting cluster are shunted by the tunnel junctions with more than two localized states, giving the principal contribution to the temperature dependence of the conductivity. In Fig. 1(c), the concentration dependences of the term $\sigma_{0}$ in Eq. (1) in intervals I and II are presented. One can see that both dependences are nearly identical. This means that temperature dependence of the conductivity in both intervals is formed in the current paths shunting the same infinite conducting cluster.

In order to check the applicability of this model the $\sigma(T)$ dependences of the molybdenum-carbon nanocomposites were fitted in the framework of the effective medium approximation [9]. The best results for the fitting were achieved by the following assumptions: the Mo-C nanocomposite was considered as a three-component mixture of the conducting and insulating species; the conducting species represent the intergrain tunnel junctions whose conductivity $\sigma_{m}$ does not depend on temperature; the dielectric domains stand for the intergrain tunnel junctions with two or three localized states. Their temperature-dependent conductivities were taken in the form $\sigma_{d 1}=\sigma_{11}\left(T / T_{0}\right)^{2.5}$, and $\sigma_{d 2}=\sigma_{12}\left(T / T_{0}\right)^{1.33}$ where the power exponents equal to 2.5 and 1.33 appear from the model [8] for the inelastic channels with $n=3$ and 2, respectively (Eq. (4)). $T_{0}$ is the characteristic temperature introduced to retain the parameters $\sigma_{11}$ and $\sigma_{12}$ in the units of conductivity. For the simplicity, its value was chosen as $1 \mathrm{~K}$. In the framework of the effective medium approximation, the conductivity of the nanocomposite $\sigma_{\text {eff }}$, the conductivities of metal $\sigma_{m}$, dielectric $\sigma_{d 1}$ and $\sigma_{d 2}$ species, metal phase fraction $X_{m}$, and dielectric phase fraction $X_{d 1}$ (3-localized states junction) are interrelated by equation [9]:

$$
X_{m} \frac{3 \sigma_{e f f}}{2 \sigma_{e f f}+\sigma_{m}}+X_{d 1} \frac{3 \sigma_{e f f}}{2 \sigma_{e f f}+\sigma_{d 1}}+\left(1-X_{m}-X_{d 1}\right) \frac{3 \sigma_{e f f}}{2 \sigma_{e f f}+\sigma_{d 2}}=1 .
$$

The model allows one to trace the evolution of the molybdenum-carbon nanocomposites' properties with increasing Mo concentration. The results of the fitting of $\sigma(T)$ dependences for the temperature intervals I and II are summarized in Fig. 2 (a), (b), where the Mo concentration evolution of phase fractions $X_{m}, X_{d 1}, X_{d 2}=1-X_{m}-X_{d 1}$, and conductivities $\sigma_{11}$, and $\sigma_{12}$ are shown. It follows from the figure that metal fraction $X_{m}$ monotonically increases while $X_{d 1}$ and $X_{d 2}$ decrease with an increase in Mo concentration (Fig. 2 (a)). The fractions either of 3-state 


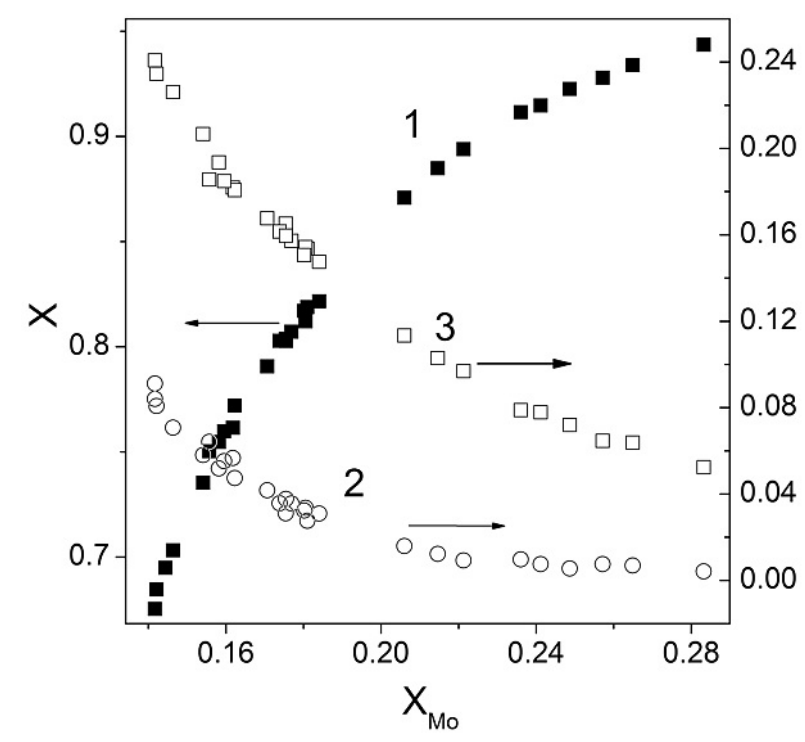

(a)

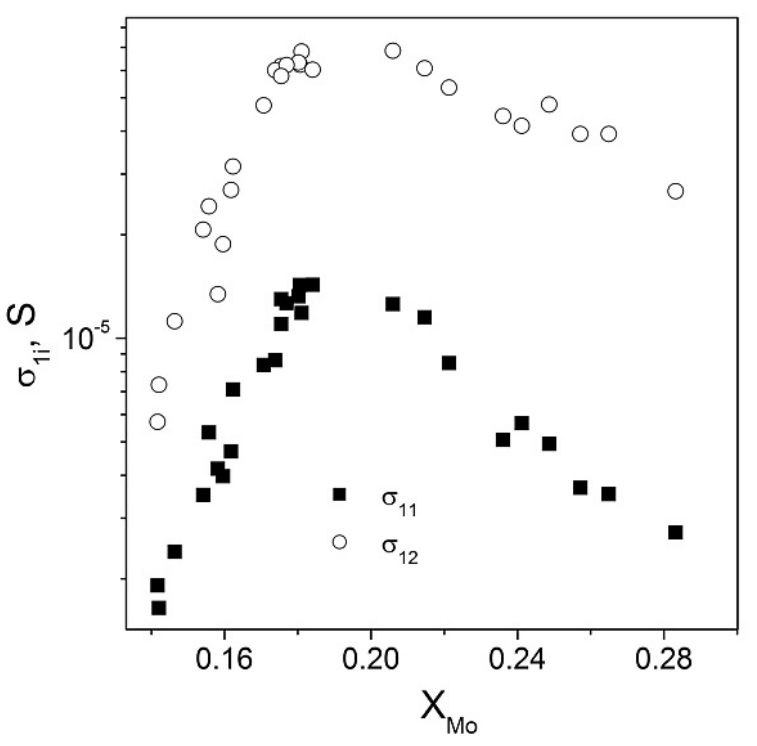

(b)

FIG. 2. Mo concentration dependences of: (a) $X_{m}$ (1), $X_{d 1}(2)$, and $X_{d 2}$ (3) phase fractions; (b) conductivities $\sigma_{11}$ and $\sigma_{12}$ of dielectric species in both temperature intervals. Explanations are given in text

and 2-state barriers remain well below the percolation threshold $(\sim 0.25)$ at Mo concentration far from the metal-insulator transition point in the metallic state $(>\sim 0.15)$. This means that the temperature-dependent conducting channels do not form the infinite conducting cluster. Thus the corrections to the conductivity of Mo-C nanocomposites are formed in the current paths consisting of the serially-connected elastic and inelastic junctions. The non-monotonic concentration behavior of $\sigma_{11}$ and $\sigma_{12}$ prefactors arises from the term $l^{n-1} \exp \{-2 l /[a(n+1)]\}$ in Eq. (4) modulated by the possible variation of the parameters of intergranular dielectric space by metal concentration increase.

\section{Conclusions}

The conductivity of the Mo-C nanocomposites was studied at $4.2-400 \mathrm{~K}$ over the molybdenum concentration range of $0.14-0.3$. The conductivity demonstrates the existence of the two distinct temperature intervals separated at temperature $20-25 \mathrm{~K}$. Within each temperature interval, the conductivity has a power character with a power exponent non-monotonically dependent on Mo content. It was shown that the model of inelastic tunneling of the electrons between conducting grains in the framework of effective medium approximation can give an adequate description of the electron transport in molybdenum-carbon nanocomposites over the entire metal concentration and temperature ranges.

\section{References}

[1] Beloborodov I.S., Lopatin A.V., Vinokur V.M., Efetov K.B. Granular electronic systems. Rev. Mod. Phys., 2007, 79, P. 469-518.

[2] Nalwa H.S. (Ed.). Handbook of Surfaces and Interfaces of Materials, Academic Press, San Diego, 2001 , Vol. 1, P. 447-508.

[3] Bozhko A., Ivanov A., Berettoni M. Chudinov S., Stizza S., Dorfman V., Pypkin B. Electroconductivity of amorphous-carbon films containing silicon and tungsten. Diamond Relat. Mater., 1995, 4, P. 488-491. 
[4] Bozhko A., Takagi T., Takeno T., Shupegin M. Electron transport in W-containing amorphous carbon-silicon diamond-like nanocomposites. J. Phys.: Condens. Matter, 2004, 16, P. 8447-8458.

[5] Efetov K.B., Tschersich A. Coulomb effects in granular materials at not very low temperatures. Phys. Rev. B, 2003, 67, P. 174205-14.

[6] Beloborodov I.S., Lopatin A. V., Vinokur V. M. Universal description of granular metals at low temperatures: Granular Fermi liquid. Phys. Rev. B, 2004, 70, P. 205120-5.

[7] Ambrosetti G., Balberg I., Grimaldi C. Percolation-to-hopping crossover in conductor-insulator composites. Phys. Rev. B, 2010, 82, P. 134201-7.

[8] Glazman L.I., Matveev K.A. Inelastic tunneling across thin amorphous films. JETP, 1988, 67, P. $1276-1282$.

[9] Vinogradov A.P. The Electrodynamics of Composite Materials (in Russian). Editorial URSS, Moscow, 2001, $208 \mathrm{p}$. 